\title{
Análise bayesiana para características de carcaça avaliadas por ultrassonografia de bovinos da raça Nelore Mocho, criados em bioma Cerrado
}

\author{
Bayesian analysis for carcass traits in Polled Nelore
}

\author{
Carina Ubirajara de Faria $^{\mathrm{I}^{*}}$ Willian Bruno Fernandes de Andrade ${ }^{\mathrm{II}}$ \\ Cristiane de Fátima Pereira ${ }^{\mathrm{I}}$ Rosiane Pereira da Silva ${ }^{\mathrm{I}}$ Raysildo Barbosa Lôbo ${ }^{\text {III }}$
}

\section{RESUMO}

Objetivou-se estimar as herdabilidades para as características de carcaça de área de olho de lombo (AOL), espessura de gordura subcutânea (EG) e espessura de gordura subcutânea na garupa (EGP8), medidas por ultrassonografia, em bovinos da raça Nelore Mocho, criados em bioma Cerrado. As informações utilizadas foram provenientes de criatórios da raça Nelore Mocho, participantes do Programa Nelore Brasil, da Associação Nacional de Criadores e Pesquisadores (ANCP), localizados nos estados que representam as maiores áreas contínuas do bioma Cerrado. As estimativas de herdabilidade foram obtidas mediante análises bi-características, sob modelo animal, utilizando a estatística bayesiana por meio do aplicativo MTGSAM. As estimativas de herdabilidade foram de média magnitude para a AOL (0,25), EG $(0,19)$ e EGP8 $(0,25)$. Os resultados obtidos sugerem que as características de carcaça, avaliadas por ultrassonografia, apresentam grande variabilidade genética e podem ser incluídas em programas de melhoramento genético de bovinos da raça Nelore Mocho.

Palavras-chave: análise de ultrassonografia, bovinos de corte características de carcaça, herdabilidade.

\section{ABSTRACT}

The purpose of this study was to estimate the genetic parameters for carcass traits of longissimus muscle area (LMA), backfat thickness $(B F)$ and rump fat thickness $(R F)$, measured by ultrasound in Polled Nelore cattle raised in the Cerrado biome. The data set was obtained from bovines Polled Nelore, proceeding from participant farms of the Nelore Brazil Program of the National Association of Breeders and Researchers, raised on pasture, from the Brazilian states that have the largest continuous areas of the Cerrado. Heritabilities were estimated by Gibbs Sampling using the MTGSAM software (Multiple Trait using Gibbs Sampler under Animal Model). The heritability estimates were 0.30, 0.17 and
0.25 for LMA, BF and RF, respectively. The results indicate that carcass traits have high genetic variability and should be included in breeding programs.

Key words: beef cattle, carcass traits, heritability, ultrasound analysis.

\section{INTRODUÇÃO}

A maior parte da carne bovina brasileira é oriunda de sistemas de produção extensivos, em regime de pastagens, e rebanhos constituídos de raças zebuínas adaptadas às condições de criação em bioma Cerrado. Das raças zebuínas, a Nelore corresponde a quase $80 \%$ do rebanho no Brasil (ABCZ, 2013). Deve-se destacar que a raça Nelore Mocho encontrase na segunda posição em número de registros pela Associação Brasileira de Criadores de Zebu (ABCZ) e, atualmente, representa $12,7 \%$ dos animais zebuínos com registro genealógico definitivo. Apesar do expressivo crescimento da raça Nelore Mocho, são poucos os estudos que avaliaram o desempenho zootécnico dessa raça.

No Brasil, o complexo agroindustrial da carne bovina caracteriza-se como sistema heterogêneo e particularidades regionais acentuadas, principalmente, nos sistemas de produção (YOKOO et al., 2008). Essa heterogeneidade acarreta problemas das mais variadas ordens, que comprometem os índices de produção do rebanho. Entretanto, o

IFaculdade de Medicina Veterinária (FAMEV), Universidade Federal de Uberlândia (UFU), Campus Umuarama, 38400-902, Uberlândia, MG, Brasil. E-mail: carinauf@famev.ufu.br. *Autor para correspondência.

IIFaculdade de Ciências Agrárias e Veterinárias (FCAV), Universidade Estadual Paulista (UNESP), Jaboticabal, SP, Brasil.

IIIAssociação Nacional de Criadores e Pesquisadores (ANCP), Ribeirão Preto, SP, Brasil. 
maior entrave é a heterogeneidade das carcaças produzidas, reflexo da pouca ou ausência de seleção para as características indicadoras de rendimento e acabamento de carcaça. De acordo com CARDOSO (2013), a falta de homogeneidade das carcaças bovinas pode contribuir para redução dos índices de exportação para a União Europeia. No entanto, a préseleção dos animais poderia auxiliar na obtenção de uniformidade das carcaças de animais abatidos.

Espera-se que uma carcaça bovina deva apresentar acabamento de gordura suficiente para garantir sua preservação e manter as características desejáveis para oconsumo. DeacordocomBOLEMAN et al. (1998), o rendimento de cortes cárneos, a porcentagem de gordura e a maciez da carne são alguns dos principais fatores que podem afetar a qualidade da carcaça. Nesse contexto, a mensuração da área de olho de lombo tem sido utilizada como indicadora do desenvolvimento muscular e, quando avaliada juntamente com medidas de espessura de gordura ou acabamento, fornecem informações relacionadas à qualidade da carcaça (PRADO et al., 2004).

Estudos científicos envolvendo estimação de parâmetros genéticos para essas características que avaliam diretamente a qualidade de carcaça (área de olho de lombo e espessura de gordura, por exemplo) em bovinos de corte, mostraram a existência de variabilidade genética para tais características, o que permite obter progresso genético por meio da seleção (FIGUEIREDO et al, 2001; YOKOO et al., 2008, LIMA NETO et al., 2009). No entanto, não há relatos na literatura sobre estimativas de parâmetros genéticos para características de carcaça, avaliadas por ultrassonografia, em bovinos da raça Nelore Mocho.

Dessa forma, faz-se necessário aplicar ferramentas de seleção direta para rendimento e acabamento de carcaça, melhorando os índices de produtividade da raça Nelore Mocho, com intuito de aumentar o progresso genético dos rebanhos e, consequentemente, a rentabilidade do setor.

Deve-se ressaltar que o método de estimação é um fator importante para obtenção dos parâmetros genéticos, juntamente com o estabelecimento de um modelo que descreva corretamente os dados. De acordo com VAN TASSEL et al. (1998), a aplicação dos modelos bayesianos em características quantitativas poderá promover rapidamente o ganho genético, devido a uma melhor identificação de genótipos superiores.

Assim, objetivou-se com este trabalho estimar os componentes de variância e parâmetros genéticos de características de carcaça, avaliadas por ultrassonografia, de bovinos da raça Nelore Mocho, criados em bioma Cerrado, utilizando a estatística bayesiana.

\section{MATERIAL E MÉTODOS}

As informações utilizadas foram provenientes de criatórios da raça Nelore Mocho, participantes do Programa Nelore Brasil, da Associação Brasileira de Criadores e Pesquisadores (ANCP), localizados nos estados de Goiás, Minas Gerais, Mato Grosso, Mato Grosso do Sul, Tocantins e Distrito Federal. Foram avaliadas as características de carcaça como área de olho de lombo (AOL), espessura de gordura subcutânea (EG) e espessura de gordura subcutânea na garupa (EGP8). As medidas de carcaça foram obtidas em animais de 330 a 610 dias de idade.

As características área de olho de lombo (AOL) e espessura de gordura subcutânea (EG) foram mensuradas entre a região da $12^{\mathrm{a}}$ e $13^{\mathrm{a}}$ costelas, transversalmente sobre o músculo Longissimus dorsi. Já a espessura de gordura subcutânea na garupa (EGP8) foi mensurada na intersecção dos músculos Gluteus medius e Biceps femoris, localizados entre o ílio e o ísquio.

Para a mensuração das características de carcaça, foram coletadas imagens de ultrassonografia por meio de um aparelho ALOKA $500 \mathrm{~V}$, com sonda linear de $17,2 \mathrm{~cm}$, de $3,5 \mathrm{MHz}$ e um acoplador acústico em conjunto com um sistema de captura de imagens (Blackbox, Bionitrics, Inc., Ames, IA, EUA). Essas imagens foram interpretadas pelo laboratório responsável pela garantia da qualidade dos dados (Aval Serviços Tecnológicos S/S). Para coleta das imagens, utilizou-se óleo vegetal e um acoplante, para garantir o contato acústico entre a sonda linear e o corpo do animal.

A estruturação dos arquivos de dados foi realizada com a utilização do programa Statistical Analysis System (SAS, 2004). Para a verificação dos efeitos de ambiente que influenciam as características morfológicas, foram utilizados os procedimentos GLM (General Linear Model) do programa SAS (SAS, 2004).

Para a formação dos grupos de animais contemporâneos, contemplaram-se os efeitos de fazenda, sexo, ano e estação de nascimento, lote de manejo aos 120, 210, 365 e 450 dias de idade. $\mathrm{O}$ efeito de estação de nascimento foi dividido em quatro classes: animais nascidos nos meses de janeiro a março (classe 1), abril a junho (classe 2), julho a setembro (classe 3) e outubro a dezembro (classe 4). Eliminaram-se os grupos contemporâneos que continham menos de quatro animais e os animais que apresentaram valores fenotípicos superiores 
ou inferiores a 3,5 desvios padrão, somados ao valor médio do seu grupo de contemporâneos.

Os componentes de variâncias e parâmetros genéticos foram estimados mediante análises uni-características e bi-características, sob modelo animal, utilizando a estatística bayesiana por meio do aplicativo Multiple Trait Gibbs Sampler for Animal Models (MTGSAM), desenvolvido por VAN TASSELL et al. (1998). Na aplicação do modelo linear misto, consideraram-se os grupos de animais contemporâneos como efeitos fixos e a idade do animal como covariável (efeito linear). Nas análises genéticas uni-características, o modelo animal pode ser representado como:

$$
y=X \beta+Z_{1} a+e
$$

Em que o $y$ é o vetor das observações, $\beta$ é o vetor dos efeitos fixos, $a$ é o vetor dos efeitos aleatórios que representam os valores genéticos aditivos diretos de cada animal, $e$ o vetor de efeitos aleatórios residuais, e $X$ e $Z_{1}$ são as matrizes de incidência que relacionam as observações aos efeitos fixos e ao efeito aleatório genético aditivo, respectivamente.

Para a realização das análises genéticas bi-características, considerou-se a medida do peso padronizado aos 120 dias de idade (P120) como "âncora", visando minimizar os efeitos da seleção, geralmente, praticada no período de desmame dos animais. Ressalta-se que se considerou 48.046 animais com informações de peso aos 120 dias de idade (P120), visto que eram animais com alguma relação de parentesco com aqueles que obtiveram medidas avaliadas por ultrassonografia de carcaça. $\mathrm{O}$ modelo completo em notação matricial é apresentado a seguir:

$$
y=X \beta+Z_{1} a+Z_{2} m+e
$$

Em que o $y$ é o vetor das observações, $\beta$ é o vetor dos efeitos fixos, $a$ é o vetor dos efeitos aleatórios que representam os valores genéticos aditivos diretos de cada animal, $m$ é o vetor dos efeitos aleatórios que representam os valores genéticos aditivos maternos (característica âncora), $e$ o vetor de efeitos aleatórios residuais, e $X, Z_{1}$ e $Z_{2}$ são matrizes de incidência que relacionam as observações aos efeitos fixos e aos efeitos aleatórios genéticos aditivos e maternal, respectivamente.

$\mathrm{Na}$ implementação da Amostragem de Gibbs, foi utilizado um tamanho de cadeia inicial de 300.000 ciclos, sendo que os primeiros 30.000 ciclos foram descartados e as amostras retiradas a cada 1.000 ciclos, totalizando 270 amostras de componentes de variância e parâmetros genéticos. As análises das amostras, da correlação serial e da convergência da cadeia de Gibbs foram realizadas com o auxílio do programa GIBANAL (VAN KAAM, 1998).
O erro de Monte Carlo foi estimado calculando a raiz quadrada da razão da variância das amostras de cada parâmetro, estimado pelo número de amostras (VAN TASSEL et al., 1998). A raiz quadrada deste valor é uma aproximação do desvio padrão do erro, associado com o tamanho da cadeia de Gibbs.

\section{RESULTADOS E DISCUSSÃO}

Os valores das médias fenotípicas para características de AOL, EG e EGP8 foram semelhantes às relatadas na literatura, considerando informações obtidas em rebanhos da raça Nelore (FIGUEIREDO et al., 2001; YOKOO et al., 2008; YOKOO et al., 2010). Ao avaliar bovinos Angus, com 14 meses de idade, SAPP et al. (2002) e KEMP et al. (2002) relataram valores superiores aos obtidos no presente estudo (Tabela 1), o que já era esperado, em virtude dos anos de seleção da raça Angus para tais características de carcaça com expressivos ganhos genéticos. De acordo com YOKOO et al. (2010), animais zebuínos têm apresentado valores menores para as médias de espessura de gordura subcutânea, comparado aos animais taurinos.

Ao avaliar os coeficientes de variação estimados, estes foram inferiores aos relatados por CREWS JR et al. (2003), YOKOO et al. (2008) e YOKOO et al. (2010), o que permite inferir sobre a adequada coleta das informações de campo utilizadas neste estudo. SAPP et al. (2002) e CREWS JR et al. (2003) avaliaram bovinos da raça Angus e Simental, respectivamente, e relataram estimativas de coeficientes de variação na ordem de 33,33\% a 46,36\% para as características de carcaça EG e EGP8, valores superiores aos obtidos neste trabalho (Tabela 1). LIMA NETO et al. (2009), ao avaliar 1.325 bovinos da raça Guzerá, também observaram maiores variações para as características relacionadas ao acabamento de carcaça (EG e EGP8). Ressalta-se que na literatura não foram encontradas informações de características de carcaça em bovinos da raça Nelore Mocho. As estimativas dos componentes de variância e parâmetros genéticos para as características de carcaça obtidas a partir de análises uni e bicaracterísticas são descritas na tabela 2. Observa-se que as estimativas de herdabilidade foram de média magnitudepara todas as características avaliadas. Esses resultados evidenciam a existência de variabilidade e a obtenção de ganho genético com a seleção direta para tais características na raça Nelore Mocho.

As estimativas de herdabilidades para as características AOL, EG e EGP8 obtidas em análises genéticas uni-características foram de $0,25,0,19$ e 0,25 , respectivamente. Ao avaliar as estimativas de herdabilidade obtidas em análises bi- 
Tabela 1 - Estatística descritiva das características peso aos 120 dias de idade (P120), área de olho de lombo (AOL), espessura de gordura subcutânea (EG) e espessura de gordura subcutânea na garupa (EGP8), de bovinos da raça Nelore Mocho, criados em bioma Cerrado.

\begin{tabular}{|c|c|c|c|c|c|c|}
\hline Característica & $\mathrm{N}^{\mathrm{o}}$ & Média & $\mathrm{DP}$ & CV (\%) & Mínimo & Máximo \\
\hline P120 (kg) & 48.046 & 126 & 20,11 & 15,93 & 54 & 222 \\
\hline $\mathrm{AOL}(\mathrm{cm})$ & 4.828 & 51,78 & 10,45 & 20,20 & 21,86 & 98,71 \\
\hline $\mathrm{EG}(\mathrm{mm})$ & 4.824 & 2,34 & 0,74 & 31,60 & 0,51 & 11,70 \\
\hline EGP8 (mm) & 4.773 & 2,86 & 1,04 & 36,46 & 0,40 & 12,10 \\
\hline
\end{tabular}

$\mathrm{DP}=$ desvio padrão $\mathrm{CV}=$ coeficiente de variação.

características, observa-se que os resultados foram semelhantes, com estimativas médias de 0,30, 0,17 e 0,25 , respectivamente. Entretanto, ao avaliar a região de credibilidade ( $95 \%$ ) da densidade posterior das estimativas de herdabilidade, observa-se que elas apresentaram menor amplitude para todas as características analisadas sob modelo animal, considerando o uso do peso aos 120 dias como característica âncora (análise bi-característica). Esse resultado indica que há efeito da seleção aplicada ao final da fase de recria, e que a inclusão do peso aos 120 dias pode auxiliar na obtenção de estimativas de maior confiabilidade.

Ao avaliar o erro de Monte Carlo, verifica-se que as estimativas de herdabilidade obtidas em análises bi-características apresentaram menores valores do erro de Monte Carlo, quando comparadas às estimativas de herdabilidade geradas em análises uni-características (Tabela 2). Entretanto, vale ressaltar que o erro de Monte Carlo foi muito pequeno para todas as análises, confirmando que o tamanho da cadeia amostral utilizada na análise bayesiana foi suficiente para obter estimativas precisas das médias das densidades posteriores. De acordo com VAN TASSEL \& VAN VLECK (1996), o erro de Monte Carlo é inversamente proporcional ao tamanho da cadeia de Gibbs. Conhecer este fator é muito importante para avaliar se a implementação da Amostragem de Gibbs foi adequada para gerar as médias posteriores das distribuições marginais dos componentes de variância e parâmetros genéticos.

$\mathrm{Na}$ figura 1, verificou-se que todas as distribuições foram semelhantes à distribuição normal que, de acordo com as pressuposições bayesianas (VAN TASSELL et al., 1998), são indicativas de convergência e obtenção de estimativas acuradas. Ressalta-se que, para todas as distribuições, os valores da média e mediana foram iguais, exceto a moda, que não foi calculada em nenhuma das distribuições (amodal).

YOKKO et al. (2008) e YOKKO et al. (2010), ao avaliar dados de carcaça de bovinos da raça Nelore, relataram estimativas similares para a característica área de olho de lombo (AOL), avaliada neste estudo. FIGUEIREDO et al. (2001) observaram valores superiores de herdabilidade, estimada em 0,41 para AOL, avaliada em bovinos da raça Nelore. Os resultados sugerem que se pode obter progresso genético para o rendimento de carcaça, utilizando as informações da área de olho de lombo (AOL), obtidas por ultrassonografia de carcaça, como critério de seleção.

Tabela 2 - Estimativas dos componentes de variância e parâmetros genéticos para área de olho de lombo (AOL), espessura de gordura subcutânea (EG) e espessura de gordura subcutânea na garupa (EGP8), em bovinos da raça Nelore Mocho, obtidas a partir de análises uni e bi-características.

\begin{tabular}{|c|c|c|c|c|c|}
\hline Característica & $\sigma_{\mathrm{a}}^{2}$ & $\sigma_{\mathrm{e}}^{2}$ & $h^{2}$ & $\begin{array}{c}\text { Região de } \\
\text { Credibilidade }(95 \%)\end{array}$ & Erro de Monte Carlo \\
\hline$A \cap I$ & 833 & 2445 & 025 & 014,035 & 00020 \\
\hline EG & 0.04 & 0.17 & 0.19 & 0.09 a 0.28 & 0.0025 \\
\hline EGP8 & 0,12 & 0,36 & 0,25 & 0,16 a 0,33 & 0,0027 \\
\hline AOL & 10,87 & 24,64 & 0,30 & 0,27 a 0,33 & 0,0010 \\
\hline EG & 0,03 & 0,17 & 0,17 & 0,13 a 0,20 & 0,0012 \\
\hline EGP8 & 0,12 & 0,36 & 0,25 & 0,22 a 0,28 & 0,0010 \\
\hline
\end{tabular}

$\sigma_{\mathrm{a}}^{2}=$ variância genética aditiva; $\sigma_{\mathrm{e}}^{2}=$ variância residual; $\mathrm{h}^{2}=$ herdabilidade.

Ciência Rural, v.45, n.2, fev, 2015. 


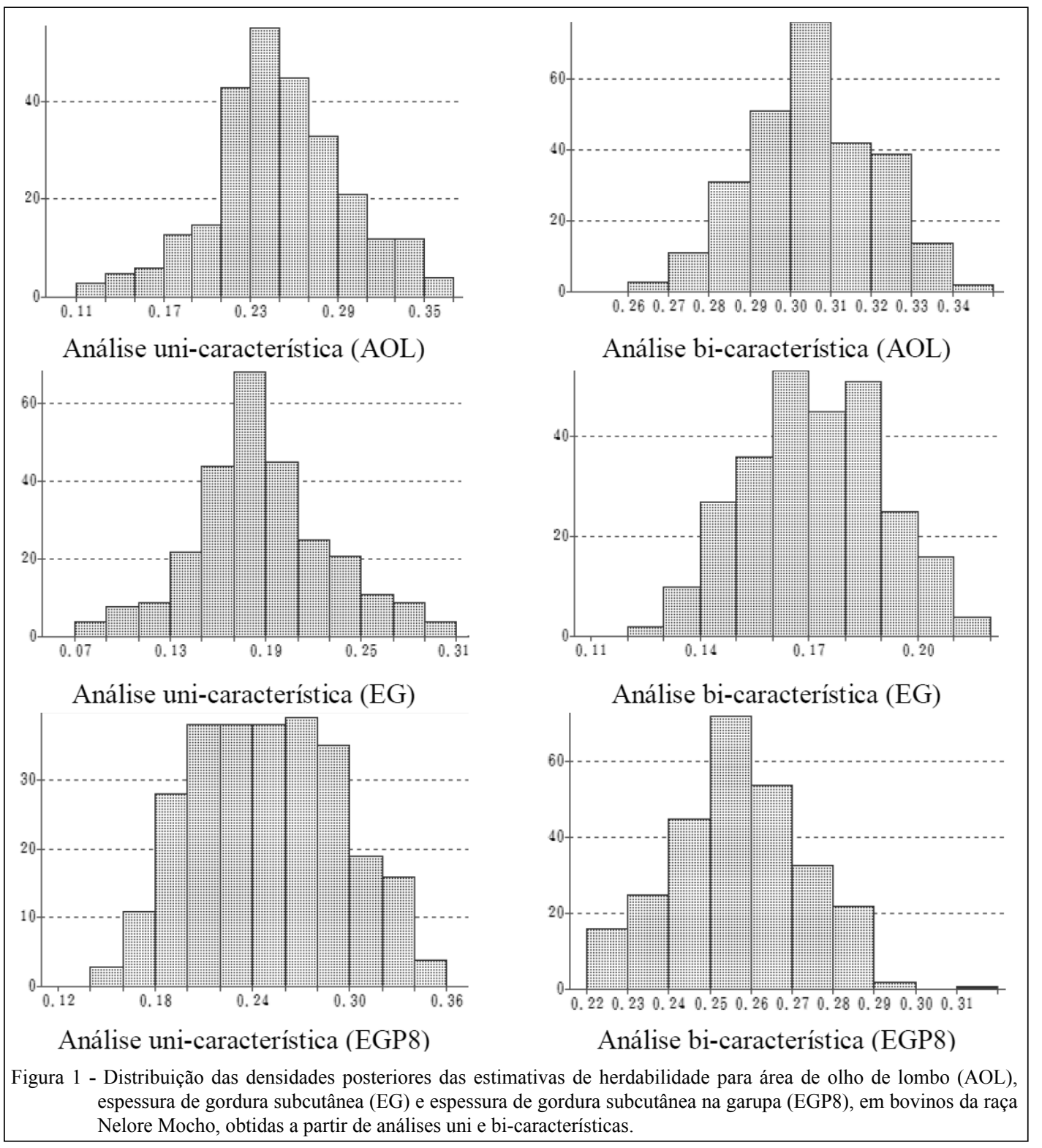

Em relação às características de acabamento de carcaça, YOKOO et al. (2008), trabalhando com dados de bovinos da raça Nelore, encontraram estimativas de herdabilidades de $0,51 \mathrm{e}$ 0,39 para a espessura de gordura subcutânea (EG) e a espessura de gordura subcutânea na garupa (EGP8), respectivamente. Neste estudo, os resultados obtidos para as características de acabamento (EG e EGP8) foram de menor magnitude, porém, sugerem que tais características são passíveis de resposta à seleção direta. Assim, infere-se que as características de carcaça, avaliadas por ultrassonografia, em bovinos da raça Nelore Mocho, podem ser incluídas em índices de seleção, que visam à obtenção de animais com maior rendimento e acabamento de carcaça. Destaca-se que não foram encontradas estimativas de herdabilidade, na literatura, para tais características, em bovinos da raça Nelore Mocho. Ressalta-se que a avaliação por ultrassonografia de carcaça é realizada por técnicos credenciados e, atualmente, a obtenção de tais informações é de fácil acessibilidade para os criatórios de bovinos de corte no Brasil.

\section{CONCLUSÃO}

As características de carcaça, avaliadas por ultrassonografia, como a área de olho de lombo (AOL), espessura de gordura subcutânea (EG) e 
espessura de gordura subcutânea na garupa (EGP8) apresentam variabilidade genética e, apesar das estimativas de herdabilidade medianas, podem ser utilizadas como critério de seleção para obtenção de progresso genético em rebanhos de bovinos da raça Nelore Mocho.

\section{AGRADECIMENTOS}

À Associação Nacional de Criadores e Pesquisadores (ANCP) pela concessão dos dados.

\section{REFERÊNCIAS}

ABCZ (ASSOCIAÇÃO BRASILEIRA DOS CRIADORES DE ZEBU). Disponível em: <http://www.abcz.org.br>. Online. Acesso em: 26 nov. 2013.

BOLEMAN, S.L. et al. National beef quality audit-1995: survey of producer-related defects and carcass quality and quantity attributes. Journal of Animal Science, v.76, p.96-103, 1998. Disponível em: $<$ http://www.ncbi.nlm.nih.gov/pubmed/9464889>. Acesso em: 28 nov. 2013. PMID: 9464889 [PubMed - indexed for MEDLINE].

CARDOSO, L.L. Estimativas do rendimento comercial de novilhos com a utilização de ultrassom. 2013. 119f. Tese (Doutorado em Zootecnia) - Programa de Pós-graduação em Zootecnia, Faculdade de Agronomia, Universidade Federal do Rio Grande do Sul, RS.

CREWS Jr, D.H. et al. Genetic parameters for carcass traits and their live animal indicators in Simmental cattle. Journal of Animal Science, v.81, p.1427-1433, 2003. Disponível em: $<$ http://www.journalofanimalscience.org/content/81/6/1427.full $>$. Acesso em: 28 nov. 2013. PMID: 12817489 [PubMed - indexed for MEDLINE]

FIGUEIREDO, L.G.G. Estimativas de parâmetros genéticos de características de carcaças feitas por ultrassonografia em bovinos da raça Nelore. 2001. 67f. Dissertação (Mestrado em Zootecnia) - Faculdade de Zootecnia e Engenharia de Alimentos, Universidade de São Paulo, Pirassununga, SP.

KEMP, D.J. et al. Genetic and environmental parameters for steer ultrasound and carcass traits. Journal of Animal Science, v.80, p.1489-1496, 2002. Disponível em: $<$ http://www.ncbi.nlm. nih.gov/pubmed/12078728>. Acesso em: 20 nov. 2013. PMID: 12078728 [PubMed - indexed for MEDLINE].
LIMA NETO, H.R. et al. Parâmetros genéticos para características de carcaça avaliados por ultrassonografia em bovinos da raça Guzerá. Arquivo Brasileiro de Medicina Veterinária e Zootecnia, v.61, p.251-258, 2009. Disponível em: <http://www.scielo. br/scielo.php?pid=S0102-09352009000100035\&script $=$ sci arttext>. Acesso em: 20 nov. 2013. doi: 10.1590/S010209352009000100035

PRADO, C.S. et al. Comparação de diferentes métodos de avaliação da área de olho de lombo e cobertura de gordura em bovinos de corte. Ciência Animal Brasileira, v.5, p.141148, 2004. Disponível em: <http://www.revistas.ufg.br/index. php/vet/article/view/26837/15357>. Acesso em: 26 nov. 2013. doi:10.5216/cab.v5i3.26837.

SAPP, R.L. et al. Effects of selection for ultrasound intramuscular fat percentage in Angus bulls on carcass traits of progeny. Journal of Animal Science, v.80, p.2017-2022, 2002. Disponível em: <http://www.animal-science.org/ content/80/8/2017.full.pdf +html>. Acesso em 2 nov. 2013. PMID: 12211367 [PubMed - indexado para MEDLINE].

SAS Institute INC. SAS ONLINEDOC ${ }^{\circledR}$ 9.1.3. Cary, NC, 2004

VAN KAAM, J.B.C.H.M. Gibanal 2.9: analyzing program for Markov Chain Monte Carlo Sequences. Wageningen Agricultural University, Wageningen, The Netherlands: Department Animal Science, 1998.

VAN TASSELL, C.P. et al. Bayesian analysis of twinning and ovulation rates using a multiple-trait threshold model and Gibbs sampling. Journal of Animal Science, v.76, p.20482061, 1998. Disponível em: <http://www.animal-science.org/ content/76/8/2048.short>. Acesso em: 1 nov. 2013. PMID: 9734854 [PubMed - indexado para MEDLINE].

YOKOO, M.J.I. et al. Genetic and environmental factors affecting ultrasound measures of longissimus muscle area and backfat thickness in Nelore cattle. Livestock Science, v.117, p.147-154, 2008. Disponível em: <http://www.sciencedirect.com/science/ article/pii/S1871141307005604>. Acesso em: 26 nov. 2013. doi:10.1016/j.livsci.2007.12.006.

YOKOO, M.J.I. et al. Genetic associations between carcass traits measured by real-time ultrasound and scrotal circumference and growth traits in Nelore Cattle. Journal of Animal Science, v.88, p.52-58, 2010. Disponível em: <http://www. journalofanimalscience.org/content/88/1/52.full.pdf + html $>$. Acesso em: 1 nov. 2013. doi: 10.2527/jas.2008-1028. 